\title{
Integral imaging acquisition and processing for visualization of photon counting images in the Mid-Wave Infrared Range
}

\author{
P. Latorre-Carmona ${ }^{\mathrm{a}}$, F. Pla ${ }^{\mathrm{a}}$, B. Javidi ${ }^{\mathrm{b}}$, \\ ${ }^{a}$ Institute of New Imaging Technologies, Universidad Jaume I Campus del Riu Sec s/n, 12071 \\ Castellón de la Plana, Spain; ${ }^{\mathrm{b}}$ Department of Electrical and Computer Engineering, University of \\ Connecticut, Storrs, CT, 06269 USA \\ Email: bahram@engr.uconn.edu
}

\begin{abstract}
In this paper, we present an overview of our previously published work on the application of the maximum likelihood (ML) reconstruction method to integral images acquired with a mid-wave infrared detector on two different types of scenes: one of them consisting of a road, a group of trees and a vehicle just behind one of the trees (being the car at a distance of more than $200 \mathrm{~m}$ from the camera), and another one consisting of a view of the Wright Air Force Base airfield, with several hangars and different other types of installations (including warehouses) at distances ranging from $600 \mathrm{~m}$ to more than $2 \mathrm{~km}$. Dark current noise is considered taking into account the particular features this type of sensors have. Results show that this methodology allows to improve visualization in the photon counting domain.
\end{abstract}

Keywords: Integral imaging, photon counting, mid-wave infrared imaging, visualization.

\section{INTRODUCTION}

Integral Imaging is a three-dimensional (3D) sensing and imaging technique with applications spanning a wide range of fields [1]-[6]. Three-dimensional reconstruction and recognition in photon counting conditions is a recent active area of research in very diverse working areas [7]-[10]. In this paper we show the applicability the maximum-likelihood estimation (MLE) method has for 3D visualization purposes on Mid-Wave Infrared (MWIR) photon counting images where the photon counting process has been applied on the elemental images. Results show that the ML method provides good visualization capabilities. This paper presents an overview of the work and some of the results shown in [11].

\section{THREE-DIMENSIONAL INTEGRAL IMAGING}

Integral imaging is a 3D imaging technique with applications in 3D profilometry and depth range estimation [12], [13]. When Integral Imaging is performed in a synthetic aperture mode, an array of sensors is used, or a sensor moving in a grid of positions [14] (see Fig. 1(a)). Three-dimensional reconstruction of images can be made considering a computersynthesized virtual pinhole array, used to inversely map the elemental images into the object space (see Fig. 1(b)). Superposition of properly shifted elemental images provides the 3D reconstructed images:

$I(x, y, z)=\frac{1}{o(x, y)} \cdot \sum_{k=0}^{K-1} \sum_{l=0}^{L-1} E_{k l}\left(x-k \frac{N_{x} \cdot p}{c_{x} \cdot M}, y-l \frac{N_{y} \cdot p}{c_{y} \cdot M}\right)$

where $I(x, y, z)$ represents the intensity of the reconstructed 3D image at depth $z, x$ and $y$ are the indexes of the pixel, $E_{k l}$ represents the intensity of the kth row and lth column elemental image, $p$ is the pitch of the cameras, $N_{x} \times N_{y}$ is the total number of pixels for each elemental image, $M=\frac{z}{f}$ is the magnification factor, $c_{x} \times c_{y}$ is the physical size of the camera sensor, and $O(x, y)$ is the overlapping number matrix.

Three-Dimensional Imaging, Visualization, and Display 2016, edited by Bahram Javidi, Jung-Young Son, Proc. of SPIE Vol. 9867, 98670N · C 2016 SPIE · CCC code: 0277-786X/16/\$18 · doi: 10.1117/12.2225872 


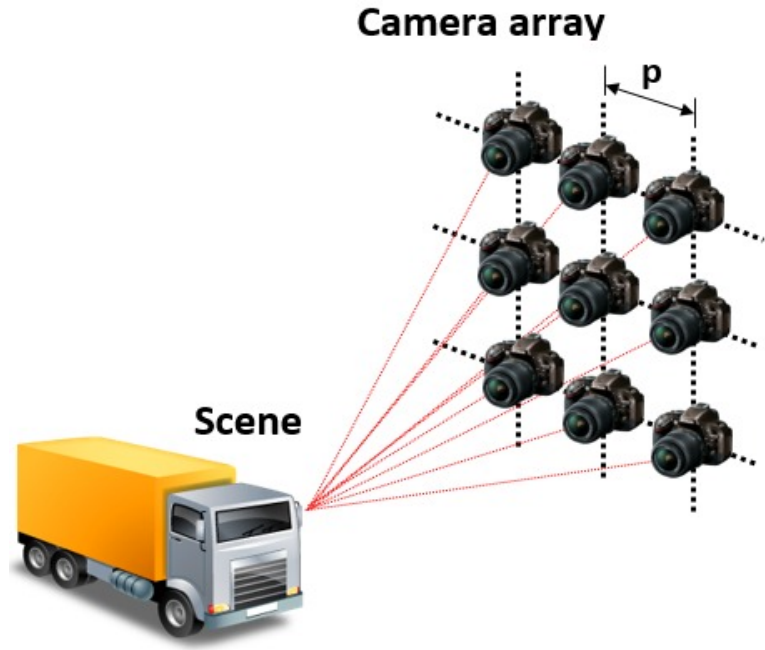

(a)

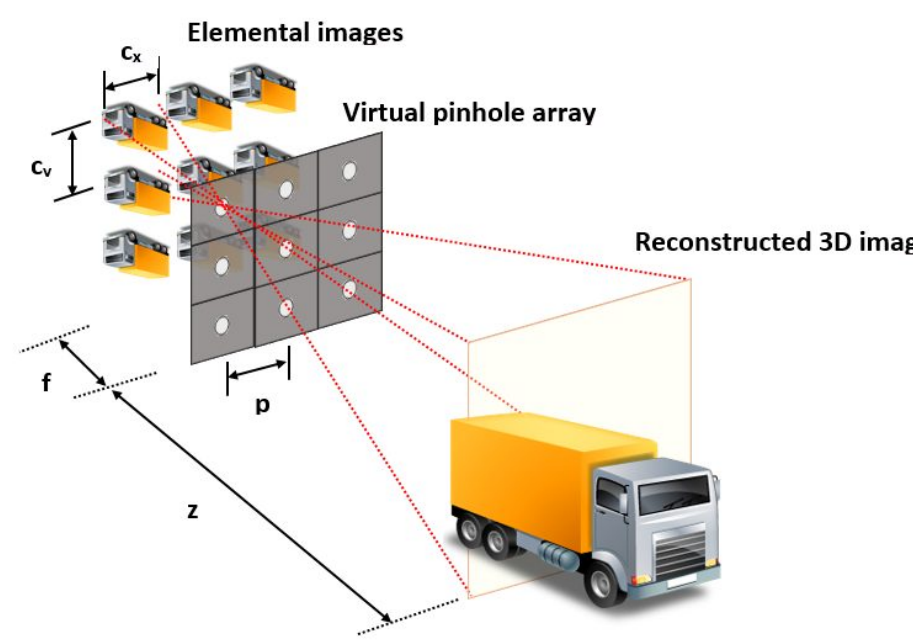

(b)

Figure 1. Synthetic Aperture Integral Imaging (SAII) acquisition and computational reconstruction method. (a) Image sensors are located in a "grid" whose spacing is given by the "pitch" parameter. (b) Optical 3D reconstruction using a virtual pinhole array and optical back-projection of the acquired elemental images.

\section{PHOTON COUNTING MODEL AND MAXIMUM LIKELIHOOD ESTIMATION FOR VISUALIZATION}

It can be shown that the integrated irradiance arriving at a camera is proportional to the mean number of photons incident onto the sensor [7], [15], and that the photon counting arrival occurrence (during a time interval) follows a Poisson density function [15]. If we consider $I_{x}$ as the spatial normalized irradiance at pixel $x$, then the Poisson distribution can be described as

$\operatorname{Pr}\left(C_{x} \mid I_{x}\right)=\frac{I_{x} C_{x \cdot e^{-I_{x}}}}{C_{x} !}$

where $C_{x}$ refers to $C$ photons at pixel $x$. On the other hand, it can be shown that, the image of an object pixel in Integral Imaging appears periodically on the elemental images in the following positions:

$\left\{\left(p+\Delta p_{k l}\right)\right\} \equiv\left\{\left(x-k \frac{N_{x} \cdot s}{c_{x} \cdot M}, y-k \frac{N_{y} \cdot s}{c_{y} \cdot M}\right)\right\}$

The irradiance of a reconstructed scene may be obtained from the photon counting elemental images ([7]) if we take into account that that the likelihood estimation for the irradiance assessment is:

$L\left(I_{p}^{Z_{0}}\right)=\prod_{k=0}^{K-1} \prod_{l=0}^{L-1} \operatorname{Pr}\left(C_{k l}\left(p+\Delta p_{k l}\right) \mid I_{p}^{z_{0}}\right)$

Considering the logarithmic expression of (4) and deriving that expression in relation to $I_{p}^{z_{0}}$ (i. e., applying a maximum likelihood strategy), one arrives at:

$\tilde{I}_{p}^{Z_{0}}=\frac{1}{N_{p} D} \cdot \sum_{k} \sum_{l} C_{k l}\left(p+\Delta p_{k l}\right)$ 
where: $D=K L / \log _{2} e$. Equation (5) is the computational reconstruction for one plane of the scene located at a specific distance using photon-counted elemental images and it is also the ML irradiance estimate of the scene at that plane. Noise sources may appear as well but if we assume they are statistically independent from the Poisson process that generates the photon counting images, then the probability term in (4) would become a product of probabilities. In that case, the partial derivative applied to Eq. (4) would cancel out the terms without dependence on $I_{p}^{Z_{0}}$ and therefore the ML estimate would remain the same. This is the assumption that has been considered in the present case.

\section{WAVELET SHRINKAGE FOR IMAGE DENOISING}

Wavelets are generated from one single function (basis function) by dilations (scaling) and translations (shifts) in time (frequency) domain. Discrete wavelet transforms (DWTs) are typically associated to Multi-Resolution Analysis (MRA), which involves the application of two sets of functions, called scaling and wavelet functions. Consider a complex scaling function and a complex wavelet. The Dual-Tree Complex Wavelet Transform (DT-CWT) [16] uses two Discrete Wavelet Transforms in parallel. The first (upper) tree gives the real part while the second (lower) gives the imaginary part of the Complex Wavelet Transform (CWT). We considered Farras filters [17] for the first decomposition stage, and Kingsburry's Q-shift filters [18], [19] for the remaining stages. Once this transform has been applied, all high frequency (MRA) coefficients that appear during this decomposition and that are lower than a particular threshold, can be converted to zero. The denoised image is obtained applying the inverse wavelet transform after this thresholding.

\section{EXPERIMENTAL RESULTS}

The elemental images were collected with a Lockheed Martin Santa Barbara Focal plane AuraSR MWIR imager from the 12th floor of the AFRL tower located at Wright Patterson Air Force Base (AFB). The camera could move in one fixed direction using a high accuracy rail apparatus. The AuraSR MWIR imager had a StingRay Optics $120 \mathrm{~mm}$ lens. The pixel size of this camera is $19.5 \mathrm{~m}$. The size of the elemental images is 10241024 pixels. Two types of scenes were acquired: (a) a scene of a road, a group of trees and a vehicle just behind one of them, and (b) a view of the airfield of Wright AFB. For (a), 8 elemental images corresponding to an acquisition in a 18 horizontal grid were considered. For (b), 10 images in a $1 \times 10$ horizontal grid were acquired (the reader is referred to [20] for further details). We simulated photon starved conditions considering a Poisson density function, which is a valid assumption in the case of Infrared detectors [21]. Three noise levels (layers) were added to each one of the elemental images for the two scenes in such a way that the total number of noise photons added was: $N_{d c}=\left\{10^{4}, 10^{5}, 10^{6}\right\}$, simulating the existence of dark current (dc) noise. An in-depth explanation about the reasoning behind these noise levels can be found in [11], [22].

Fig. 2(a) shows one of the airfield scene elemental images under normal illumination conditions. In that scene, the hangars are at a distance $960 \mathrm{~m}$ and the deposit is at a distance of around $2.2 \mathrm{Km}$. Fig. 2(b) shows one of the trees and car scene elemental images under normal illumination conditions, as well. The trees in front of the car are at a distance of approximately $210 \mathrm{~m}$, and the vehicle is at a distance of $237 \mathrm{~m}$. Figs. 2(c)-(f) show the reconstructed scene for the airfield and the trees and car scene, for $2.2 \mathrm{Km}$ and $237 \mathrm{~m}$ distance, respectively. Figs. 2(c)-(d) show the case of the airfield scene with $\left\{N_{p}, N_{d c}\right\}=\left\{3.0 \times 10^{5}, 0\right\}$ and $\left\{N_{p}, N_{d c}\right\}=\left\{3.0 \times 10^{5}, 1.0 \times 10^{6}\right\}$ photons, respectively. Figs. 2(e)-(f) show the case of the car and trees scene with $\left\{N_{p}, N_{d c}\right\}=\left\{3.0 \times 10^{5}, 0\right\}$ and $\left\{N_{p}, N_{d c}\right\}=\left\{3.0 \times 10^{5}, 1.0 \times 10^{6}\right\}$ photons, respectively. In order to help improve its visualization, wavelet shrinkage technique for image denoising (explained in Section 4 but whose particular details can be found in [11]) was applied on the depth reconstructed images. For the car and trees scene, a threshold value of $T=4$ was used. For the airfield scene, a threshold value of $T=20$ was considered.

\section{CONCLUSIONS}

In this paper, we have summarized our work made for the visualization enhancement of 3D scenes in photon starved environments corresponding to Mid-Wave Infrared 3D data of real scenes ([11]). We have shown that visualization improves using multi-perspective photon-counted images, in relation to the information provided by each photon-counted elemental image. 


\section{ACKNOWLEDGMENTS}

The authors greatfully acknowledge financial support from project PROMETEOII/2014/062 funded by the Generalitat Valenciana (regional government). Authors also acknowledge Dr. Daniel LeMaster, Air Force Research Laboratory, Wright Patterson Air Force Base, Ohio for the integral images used in [11] and in this paper, and also for his very helpful feedback about the MWIR camera used for the acquisition of these images.

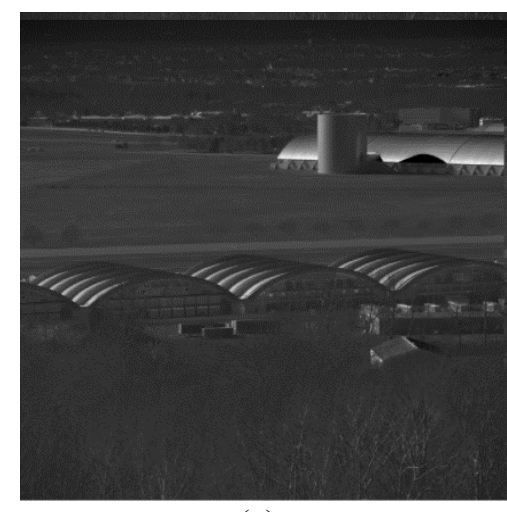

(a)

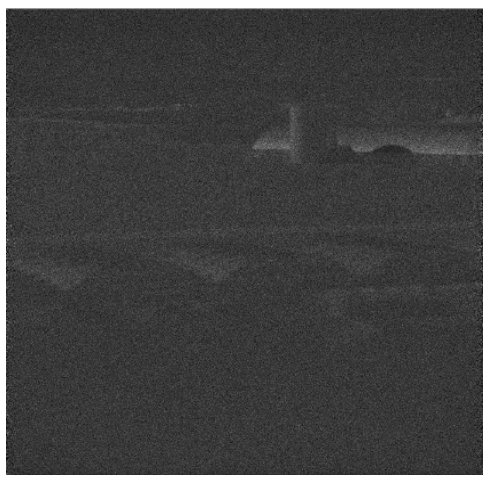

(d)

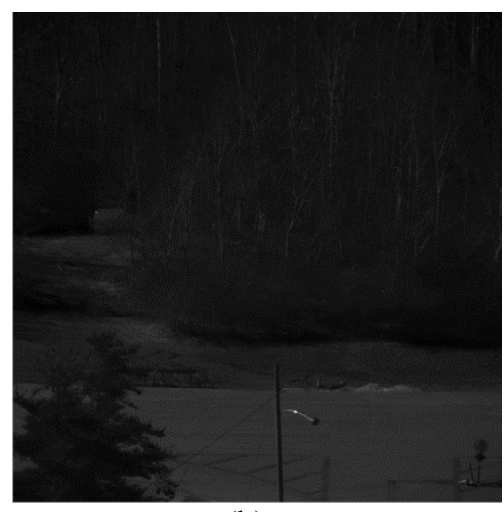

(b)

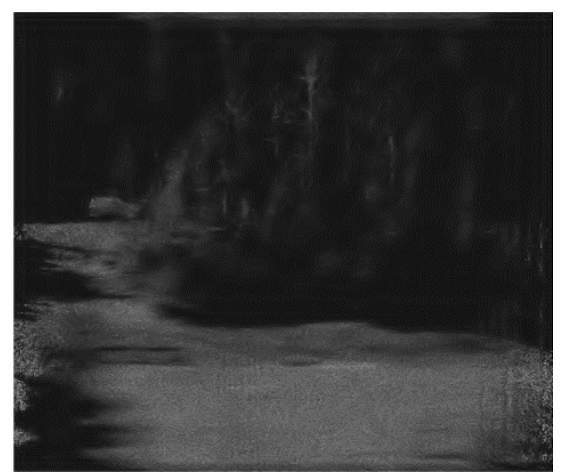

(e)

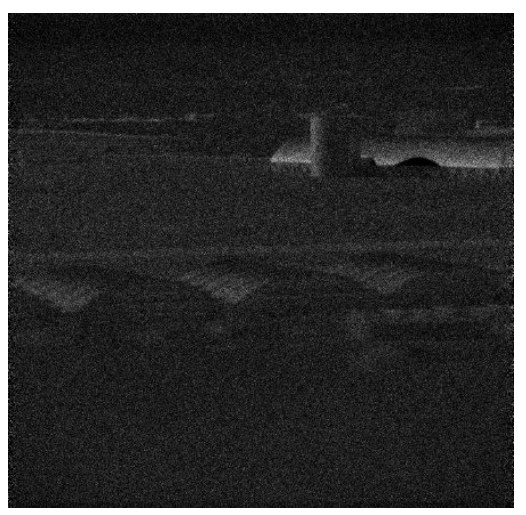

(c)

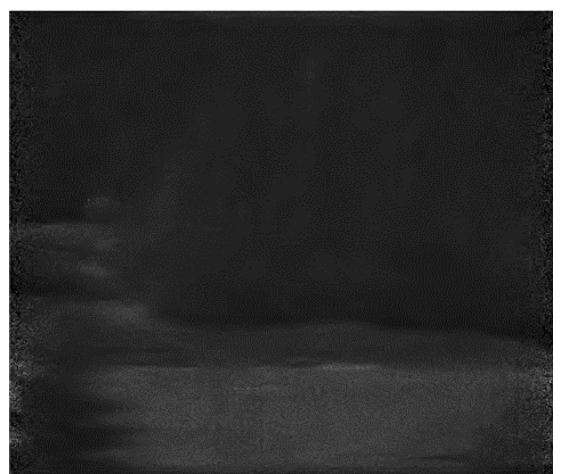

(f)

Figure 2. Reconstructed images. (a) Airfield scene elemental image. (b) Car scene elemental image. (c) Reconstructed airfield scene at $2.2 \mathrm{Km}$ with $N_{p}=3.0 \times 10^{5}$ photons and $N_{d c}=0$. (d) Reconstructed airfield scene at $2.2 \mathrm{Km}$ with $N_{p}=3.0 \times 10^{5}$ photons and $N_{d c}=1.0 \times 10^{6}$ photons. (e) Reconstructed car scene at $237 \mathrm{~m}$ with $N_{p}=3.0 \times 10^{5}$ photons and $N_{d c}=0$. (f) Reconstructed car scene at $237 \mathrm{~m}$ with $N_{p}=3.0 \times 10^{5}$ photons and $N_{d c}=1.0 \times 10^{6}$ photons (all of them originally appeared in [11]).

\section{REFERENCES}

[1] A. Stern and B. Javidi, “3-D image sensing, visualization, processing using integral imaging," Proc. IEEE, vol. 94, no. 3, pp. 591-607, Mar. 2006.

[2] B. Javidi, F. Okano, and J. Y. Son, Three-Dimensional Imaging, Visualization, Display. Berlin, Germany: Springer, 2009.

[3] R. Martinez-Cuenca, G. Saavedra, M. Martinez-Corral, and B. Javidi, "Progress in 3-D multiperspective display by integral imaging," Proc. IEEE, vol. 97, no. 6, pp. 1067-1077, Jun. 2009. 
[4] M. Cho and B. Javidi, "Three-dimensional visualization of objects in turbid water using integral imaging," J. Display Technol., vol. 6, no. 10, pp. 544-547, Oct. 2010.

[5] J. Arai et al., "Integral three-dimensional television using a 33-megapixel imaging system," J. Display Technol., vol. 6, no. 10 , pp. $422-430$, Oct. 2010.

[6] F. Okano, J. Arai, K. Mitani, and M. Okui, "Real-time integral imaging based on extremely high resolution video system," Proc. IEEE, vol. 94, no. 3, pp. 490-501, Mar. 2006.

[7] B. Tavakoli, B. Javidi, and E. Watson, "Three-dimensional visualization by photon counting computational integral imaging,” Opt. Exp., vol. 16, no. 7, pp. 4426-4436, 2008.

[8] S. Yeom, B. Javidi, and E. Watson, "Photon counting passive 3D image sensing for automatic target recognition," Opt. Exp., vol. 13, pp. 9310-9330, 2005.

[9] S. Yeom, B. Javidi, and E. Watson, "Three-dimensional distortion-tolerant object recognition using photon-counting integral imaging,” Opt. Exp., vol. 15, pp. 1513-1533, 2007.

[10] M. DaneshPanah, B. Javidi, and E. A. Watson, "Three dimensional object recognition with photon counting imagery in the presence of noise," Opt. Exp., vol. 18, pp. 26450-26460, 2010.

[11] P. Latorre-Carmona, D. LeMaster, B. Javidi, "Three-Dimensional Visualization of Long Range Scenes by Photon Counting Mid-Wave Infrared Integral Imaging”, IEEE/OSA Journal of Display Technology, vol. 11, pp. 908-912, 2015.

[12] M. DaneshPanah, B. Javidi, "Profilometry and optical slicing by three-dimensional imaging", Optics Letters, vol. 34, pp. 1105-1107, 2009.

[13] S. H. Hong, J. S. Jang, and B. Javidi, "Three-dimensional volumetric object reconstruction using computational integral imaging,” Opt. Exp., vol. 3, pp. 483-491, 2004.

[14] J. S. Jang and B. Javidi, "Three-dimensional synthetic aperture integral imaging," Opt. Lett., vol. 27, pp. 11441146, 2002.

[15] J. W. Goodman, Statistical Optics. New York, NY, USA: Wiley, 1985.

[16] N. G. Kingsbury, "The dual-tree complex wavelet transform: A new efficient tool for image restoration and enhancement," in Proc. Eur. Signal Process. Conf., 1998, pp. 319-322.

[17] F. Abdelnour and I. W. Selesnick, "Design of 2-band orthogonal near symmetric CQF," in Proc. IEEE Int. Conf. Acoust., Speech Signal Process., Salt Lake City, UT, USA, 2001, vol. 6, pp. 3693-3696.

[18] N. Kingsbury, "A dual-tree complex wavelet transform with improved orthogonality and symmetry properties," in Proc. IEEE Int. Conf. Image Process., 2000, vol. 2, pp. 375-378.

[19] M. Vetterli and J. Kovacevic, Wavelets and Subband Coding. Englewood Cliffs, NJ, USA: Prentice-Hall, 1995.

[20] D. LeMaster, B. Karch, and B. Javidi, "Mid-wave infrared 3D integral imaging at long range,” J. Display Technol., vol. 9, no. 7, pp. 545-551, Jul. 2013.

[21] E. L. Dereniak and G. L. Boreman, Infrared Detectors and Systems, ser. Pure Appl. Opt.. New York, NY, USA: Wiley, 1996.

[22] J. W. Beletic et al., "Teledyne Imaging Sensors: Infrared imaging technologies for astronomy and civil space," Proc. SPIE, vol. 7021, High Energy, Optical, Infrared Detectors for Astronomy III, pp. 1-14, 2008. 